\title{
Long-term radio behaviour of GPS sources and candidates
}

\author{
M. Tornikoski ${ }^{1, \star}$, I. Torniainen ${ }^{1}$, A. Lähteenmäki ${ }^{1}$, T. Hovatta ${ }^{1}$, E. Nieppola ${ }^{1}$, M. Turunen ${ }^{1}$, \\ M. Lainela ${ }^{2}$, E. Valtaoja ${ }^{2,3}$, M.F. Aller ${ }^{4}$, H.D. Aller ${ }^{4}$, M. Mingaliev ${ }^{5}$, and S. Trushkin ${ }^{5}$ \\ 1 Metsähovi Radio Observatory, Helsinki University of Technology TKK, Metsähovintie 114, 02540 Kylmälä, Finland \\ 2 Tuorla Observatory, University of Turku, Väisälantie 20, 21500 Piikkiö, Finland \\ 3 Department of Physics, University of Turku, 20100 Turku, Finland \\ 4 Department of Astronomy, University of Michigan, Ann Arbor, MI, 48190, USA \\ 5 Special Astrophysics Observatory, RAS, Nizhnij Arkhyz, Karachaevo-Cherkesia, 369167 Russia
}

Received 2008 Dec 8, accepted 2008 Dec 18

Published online 2009 Feb 15

Key words galaxies: active - quasars: general - radio continuum: galaxies

This paper is a summary of the work that our group has done (and recently published in several papers) on long-term radio variability of GPS sources. We have studied the long-term (up to 30 years) variability of GPS sources and candidates, with emphasis on the high-frequency radio domain. Our data sets show that only a relatively small number of these sources retain their convex spectra when they are monitored densely and for long periods of time. The current GPS samples are especially contaminated by small, beamed blazar-type sources. Also the remaining population with consistently convex GPS-type spectra seems to be heterogeneous, falling into several subpopulations when their observed properties are used for clustering them through a self-organizing map.

\section{Introduction}

Since the end of the 1970's our team has studied Active Galactic Nuclei (AGNs) at high radio frequencies (22 to 90 $\mathrm{GHz}$ ) at Metsähovi Radio Observatory in Finland (Salonen et al. 1983, 1987; Teräsranta et al. 1987, 1992, 1998, 2004; Nieppola et al. 2007). In addition to our Metsähovi observing projects, we have observed southern to equatorial AGNs with the Swedish-ESO Submillimetre Telescope (SEST) in Chile from 1988 to 2003, i.e., until the decommissioning of SEST (e.g., Tornikoski et al. 1996, 1999, 2000, 2001, 2002). Our main focus has been on the high-frequency radio variability of AGNs, but sometimes we have also carried out one to a few epoch observations of AGNs that have earlier not been observed at high radio frequencies.

During our observing projects we realized that some sources that were earlier assumed to be very faint at high radio frequencies and thus usually excluded from high-frequency studies, could in times be much brighter than expected. The fact that at least some of the sources turned out to be bright in the millimetre-domain could be due to either variability, or to an unusual continuum spectrum peaking at relatively high frequencies. In either case, it was possible that lots of interesting, potentially very bright sources had been excluded from high-frequency radio studies. If at least some of them belonged to the extreme-peaking GPS population, maybe the number of high-peaked sources would turn out to be larger than earlier assumed.

\footnotetext{
^ Corresponding author: Merja.Tornikoski@tkk.fi
}

When samples for high-frequency studies are selected by using low-frequency data only, the population of sources that peak above ca. $10 \mathrm{GHz}$ could be strongly underrepresented. Also the very low number of data points in surveys may play a role in the selection process. Selecting GPS source candidates based on one-epoch observations is problematic if the source is variable, because it is impossible to determine whether the source spectrum has a consistently GPS shape or whether the source was only observed in a very active state and its spectrum was temporarily inverted. In many radio-loud quasars, the spectrum at high radio frequencies flattens considerably or even becomes inverted during an outburst. In addition, when the GPS-candidate selection for higher frequency studies is made using catalogues with data entries possibly more than a decade older than the high-frequency observations, the non-simultaneity of the observations makes it impossible to determine the true shape of the radio spectrum.

\section{Results from the first studies}

In Tornikoski et al. (2000) we found indications that a large number of sources with high peak frequencies may exist. The existence of such sources is easily ignored, because extreme peaking sources are not systematically searched for at high radio frequencies, and most of the GPS catalogues are based on data taken at relatively low frequencies. Therefore we wanted to investigate the sources that we had been observing in Metsähovi and SEST in more detail in order 
to see if we would be able to identify new high-peaked sources.

Our initial source sample consisted of ca. 200 radiobright AGNs that had been monitored in Metsähovi, or with SEST, or both, at several radio bands in the frequency range of 22 to $230 \mathrm{GHz}$. The data streams were from 15 to ca. 25 years long. In addition to using our own observational data base, we also searched for additional radio data from the literature, see Tornikoski et al. (2001) for the complete list of references.

Using these data sets, we plotted multifrequency continuum spectra in order to search for new GPS-type sources and especially extreme-peaked sources (Tornikoski et al. 2001). We also wanted to study our source sample in order to see whether we could confirm existing GPS classifications for sources that were identified as GPS sources in the literature (de Vries et al. 1995, 1997; Tingay et al. 1997; Stanghellini et al. 1998; Dallacasa et al. 1998; O'Dea 1998).

12 out of the 14 sources that had been classified as GPS sources or candidates in the literature showed flux density variability resembling that of ordinary flat-spectrum quasars. For six of them, the average shape of the continuum spectrum was steep or flat after the inclusion of our new data. We also identified 12 new sources with convex spectra, but most of them were variable and thus contradicting the classical definition of GPS sources.

\section{Next step: a closer look at the "genuine" GPS sources}

In the studies we made in Tornikoski $(2000,2001)$ it turned out that most of the sources from our own monitoring sample, in spite of some of them at least at times having convex spectra, did not adhere to the classical GPS source definition due to their variability and temporal changes in the continuum spectra. This was also true for sources that had been classified as GPS sources/candidates in the literature (e.g., some sources from de Vries et al. 1995, 1997; Stanghellini et al. 1998; Dallacasa et al. 1998; and O'Dea 1998). Among the ones that we had observed in the highfrequency domain, there were no nonvariable quasar-type peaked-spectrum sources.

Initially our own source samples had been formed by the desire to monitor sources that were somehow "interesting", i.e. known to be or supposed to be variable, at least in some frequency domain. It was possible that our samples included the incorrectly classified, variable GPS source candidates, but the rest of the sources classified as GPS sources in the literature would more persistently adhere to the canonical GPS definition. Therefore we formed two new GPS source samples. One of them includes 60 sources, mostly quasar-type, classified as GPS sources or GPS source candidates in the literature. For the full source list and literature references, see Torniainen et al. (2005). This sample also includes the candidate GPS sources - even though already known to be relatively variable - from Tornikoski et al. (2000, 2001). As a comparison sample we constructed a list of 96 GPS galaxies, also identified as such in the literature. For the full source list and literature references for this sample, see Torniainen et al. (2007).

We made new observations of these sources at $37 \mathrm{GHz}$ in Metsähovi, at $90 \mathrm{GHz}$ with the SEST, and we obtained multifrequency 1-22 GHz data taken with the RATAN-600 telescope in Russia. Additionally, we obtained unpublished data from the University of Michigan Radio Observatory (UMRAO) at 4.8, 8.0 and $14.5 \mathrm{GHz}$ (see Aller et al. (1985) for description of the observing system). In addition to these new observational data, we also searched for complementary radio data from the literature, see Torniainen et al. $(2005,2007)$ for the complete list of references. The longest data streams were of the order of 30 years, but at some frequency bands there were only one or very few data points.

With the long-term multifrequency spectra at hand it was evident that among the 16 sources - all of them of the quasar-type - that we had expected to possibly turn out to be "new GPS sources", not a single one could be classified as such. Most of them were highly variable flatspectrum sources that showed convex spectra only during active states, and even the ones whose spectra remained somewhat convex during the various states of activity were highly variable especially in the high-frequency domain $(>22 \mathrm{GHz})$. Furthermore, the majority of sources classified as GPS sources in the literature did not retain their GPS spectrum. Only five out of 44 GPS sources had a consistently convex spectum (with their spectral index below the turnover, $\alpha_{\text {below }}>0.5$ ) and little variability (fractional variability index $V<3$ ); see Torniainen et al. (2005) for a more thorough discussion on the classification criteria. Two of them are galaxies, two are low-polarization quasars, and for one of them no optical identifications are available.

With the new data, the remaining 39 sources earlier classified as GPS sources now turned out to be variable sources, many of them having inverted spectra only during prominent activity stages. Also many of the sources which still had a convex spectrum were at times highly variable. Thus our main conclusion for this sample was that number of genuine GPS sources, with consistently convex spectra and none to modest variability, is much lower than earlier assumed.

For our comparison sample of 96 GPS galaxies we unfortunately had much sparser data sets than for the abovementioned quasar-dominated sample. Many of them were included in our source list only when we started this project. Thus for some of them we only had few-epoch observations, and for practically none of them we had such extensive flux history as for the GPS quasars. Due to the sparseness of data it was difficult or impossible to determine the actual spectra for ca. $30 \%$ of the sources. For the rest of them we had enough data for determining the shape of the continuum spectra, and the number of sources retaining their GPS spectra turned out to be higher than for quasars. 29 out of 96 sources classified as GPS galaxies in the literature retained 
a convex spectrum. However, also steep and flat spectra, as well as convex spectra with high variability were found.

Thus there are indications that the number of genuine GPS galaxies is higher than that of GPS quasars. However, there seem to be several incorrect classifications among them as well. Additionally, because our data streams for these sources were in general much shorter than for the quasar population it may turn out that some of the galaxies now showing convex spectra will not retain their spectral shape when observed for a longer period of time.

\section{Long-term variability: what have we learnt?}

Many of the early GPS source samples in the literature have been produced by first combining data points from different catalogues originating from different epochs, and then by selecting sources with convex radio spectra to be classified as GPS sources. At times single-epoch multifrequency spectra or observations spanning a couple of years have been used. This approach easily generates very heterogeneous samples, since a peaked spectrum can be produced by several different effects. Typical cases producing misclassified sources are variable flat-spectrum sources having been observed when one flaring component dominates the high-frequency part of the spectrum and creates a temporarily inverted spectrum that can last for months, or in extreme cases even years.

Our group has studied the long-term variability of AGNs at high radio frequencies in detail (e.g., Hovatta et al. 2007, 2008a, 2008b). Our findings stress the importance of longterm monitoring for fully understanding the source variability behaviour or for defining continuum spectra. In our sample of radio-bright, variable AGNs, large flares are seen on average every four to six years, and they typically last 2.5 years at 22 and $37 \mathrm{GHz}$, but can range between 0.3 to 13.2 years. During the time between the large flares these sources may exhibit smaller-scale variability or the base flux density level may gradually change, or both. Nevertheless, the truly prominent flares occur relatively rarely, and typically last for at least a few years even in the $37 \mathrm{GHz}$ frequency band.

Due to the somewhat limited access to radio telescopes one is easily tempted to draw conclusions about the source behaviour based on sparse data sets, or sometimes just on one-epoch data. Our findings show that long time series are more important than very dense sampling, and that even three to five years of data may not be enough for revealing the "typical behaviour" of a source. With too short data sets incorrect conclusions are easily drawn about the variability, the shape of the continuum spectrum, the SED, the detection probability at high frequencies, and so on. This leads to misinterpretation of source types and subtypes in samples severely contaminated by incorrectly classified sources, as we believe has happened in the case of the GPS population.

\section{Cluster analysis of GPS sources and candidates}

Except for the spectral shape there seemed to be no clear and simple "GPS source type properties" in the sources that we classified as genuine GPS sources in Torniainen et al. $(2005,2007)$. There are both quasars and galaxies, compact symmetric objects (CSOs) and core-jet morphologies, variable and non-variable sources. Intrigued by this variety of objects still classified as GPS sources, and seeking for understanding of their physics, we wanted to study their properties by using an advanced analysis method (Torniainen et al. 2008). We used a self-organized map (SOM), which is an unsupervised neural network that uses no a priori assumption of the cluster memberships. Using a large collection of parameters, the algorithm places the objects on a multidimensional map so that the Euclidian distance of the parameter vectors of similar objects is minimized. One of the advantages of this method is its ability to analyse incomplete data matrices, i.e. the method can be effectively used even when some data are missing for some sources.

We used a sample of 206 GPS and high frequency peaker (HFP) sources for this detailed study of the underlying populations of various source types. For completeness, the sample included basically all sources that have been identified as GPS sources or candidates in the literature. Thus the sample included also sources that during our previous studies (Sects. 2 and 3) turned out not to be good representatives of the genuine GPS source type, i.e., also sources whose spectra did not retain a convex shape or sources that exhibited considerable variability. The classification information from our earlier studies was not used during the clustering process, but only for studying the source clusters after the maps had been produced and to see to what degree the outcome would agree with our earlier findings.

The parameters that we used for the analysis were collected from our own observations as well as from the literature, including: redshift, size, radio power, optical and radio polarization information, optical magnitudes at various wavebands, optical colours, hydrogen column density, power law slope in X-rays, radio variability indices, and various parameters describing the shape of the radio spectrum. For tracing the locations of different types of sources on the maps we also used auxiliary classification information from our previous papers (for the spectral classification of GPS sources and candidates) and from the literature (e.g., for optical identification and VLBI morphology).

We tested several clustering methods and clustering parameters. There were no substantial differences between the various approaches, even though for some single sources the cluster memberships change when changing the clustering method or the parameter space. For the "big picture" the outcome of the various runs remained consistent: when combining the cluster map and the auxiliary identification information, the map could be divided roughly into four quarters. Two of them are populated with quasar-type sour- 
ces, one of them with consistently GPS-type spectra and the other with other types of spectra. In one half of the map there are mainly galaxies; again one side is dominated by consistently GPS-type spectra, while the other side has other types of spectra (flat, steep, convex with variability, or those with too few data points for accurate determination of their spectra). As they are not numerical quantities, neither the optical identification, VLBI morphology or our spectral classification were used by the algorithm, and therefore the formation of groups of sources similar in these properties is likely to reflect some genuine similarities between the sources.

Our results confirm the contamination of GPS samples by small, beamed blazar-type sources. More than one quarter of the map is populated by variable flat-spectrum sources and variable sources that have inverted spectra only during outbursts. These sources should be excluded from GPS samples when new "master lists" for GPS sources are being constructed.

Sources with confirmed GPS-type spectra form various separate clusters, and it seems likely that there are diverse subpopulations of GPS sources in addition to the quasar vs. galaxy dualism. Our analyses produce a cluster of very young (confirmed by kinematic age estimates) galaxy CSOs with rather low radio powers and - somewhat unexpectedly - low intrinsic turnover frequencies. There is another cluster consisting of young CSO and compact double (CD) quasars and galaxies with high peak frequencies and high radio powers, and thus considerably different from the aforementioned cluster. We have also identified a cluster that may represent free-free absorbed sources, as well as another one of quasars and galaxies with mostly core-jet morphologies and consistent GPS-type spectra. In order to really understand the fundamental differences between the various GPS subpopulations, a more thorough detailed multiwaveband approach is needed. In particular, more data are needed on the sizes, age estimates, gas content and distribution etc. to tackle the question of the physical differences of the underlying populations.

\section{Conclusions}

We have studied the long-term (up to 30 years) variability of GPS sources and candidates, with emphasis on the otherwise sparsely studied high-frequency radio domain.

It turned out that many of the sources classified as GPS sources in the literature do not have a consistently convex spectrum, and furthermore, many of them are highly variable. Most of the GPS source lists in the literature are based on too few data points, and often also on non-simultaneous data, leading to incorrect classifications.

If one wants to study the "true" GPS source population, i.e. sources that retain a convex spectrum and show relatively little time variability, a new master list based on our findings should be used instead of those given in the earlier literature, where the inclusion of blazars and other variable flat-spectrum sources contaminates the GPS source lists. In particular, sources classified as flat-spectrum sources or as of the "inverted during burst"-type in Torniainen et al. (2005, 2007) clearly stand out as sources that have earlier been misclassified, probably due to too sparse data. With long-term multifrequency data at hand, their spectra no longer resemble those of typical GPS sources.

Our cluster analysis through self-organizing maps showed that also the remaining sources with consistent GPS spectra are otherwise heterogeneous and do not form one single population. Even the quasar vs. galaxy dualism is not sufficient to explain the persistent division into several subclusters. Even though most of the source properties of the genuine GPS sources are in accordance with the "youth scenario", other subpopulations are also probable.

Acknowledgements. The authors acknowledge the support of Academy of Finland to the Metsähovi and SEST observing projects. UMRAO is supported in part by funds from the NSF and by funds from the University of Michigan Department of Astronomy. The authors made use of the database CATS (Verkhodanov et al. 1997) of the Special Astrophysical Observatory. This research made use of the NASA/IPAC Extragalactic Databse (NED), which is operated by the Jet Propulsion Laboratory, California Institute of Technology, under the contract with the National Aeronautics and Space Administration.

\section{References}

Aller, H.D., Aller, M.F., Latimer, G.E., Hodge, P.E.: 1985, ApJS 59,513

Dallacasa, D., Bondi, M., Alef, W., Mantovani, F.: 1998, A\&AS 129,219

Hovatta, T., Tornikoski, M., Lainela, M., Lehto, H.J., Valtaoja, E., Torniainen, I., Aller, M.F., Aller, H.D.: 2007, A\&A 469, 899

Hovatta, T., Nieppola, E., Tornikoski, M., Valtaoja, E., Aller, M.F., Aller, H.D.: 2008a, A\&A 485, 51

Hovatta, T., Lehto, H.J., Tornikoski, M.: 2008b, A\&A 488, 897

Nieppola, E., Tornikoski, M., Lähteenmäki, A., et al.: 2007, AJ 133, 1947

O'Dea, C.P.: 1998, PASP 110, 493

Salonen, E., Lehto, H., Urpo, S., et al.: 1983, A\&AS 51, 47

Salonen, E., Teräsranta, H., Urpo, S., et al.: 1987, A\&AS 70, 409

Stanghellini, C., O'Dea, C.P., Dallacasa, D., Baum, S.A., Fanti, R., Fanti, C.: 1998, A\&AS 131, 303

Teräsranta, H., Valtaoja, E., Haarala, S., et al.: 1987, A\&AS 71, 125

Teräsranta, H., Tornikoski, M., Valtaoja, E., et al.: 1992, A\&AS 94,121

Teräsranta, H., Tornikoski, M., Mujunen, A., et al.: 1998, A\&AS 132,305

Teräsranta, H., Achren, J., Hanski, M., et al.: 2004, A\&A 427, 769

Tingay, S.J., Jauncey, D.L., Reynolds, J.E., et al.: 1997, AJ 113, 2025

Torniainen, I., Tornikoski, M., Teräsranta, H., Aller, M.F., Aller, H.D.: 2005, A\&A 435, 839

Torniainen, I., Tornikoski, M., Lähteenmäki, A., Aller, M.F., Aller, H.D., Mingaliev, M. G.: 2007, A\&A 469, 451

Torniainen, I., Tornikoski, M., Turunen, M., et al.: 2008, A\&A 482,483

Tornikoski, M., Valtaoja, E., Teräsranta, H., et al.: 1996, A\&AS 116,157 
Tornikoski, M., Tingay, S.J., Mücke, A., et al.: 1999, AJ 118, 1161 Tornikoski, M., Lainela, M., Valtaoja, E.: 2000, AJ 120, 2278

Tornikoski, M., Jussila, I., Johansson, P., Lainela, M., Valtaoja, E.: 2001, AJ 121, 1306

Tornikoski, M., Lähteenmäki, A., Lainela, M., Valtaoja, E.: 2002, ApJ 579, 136
Verkhodanov, O.V., Trushkin, S.A., Andernach, H., Chernenkov, V.N.: 1997, in: G. Hunt, H.E. Payne (eds.), Astronomical Data Analysis Software and Systems VI, ASPC 125, p. 322

de Vries, W.H., Barthel, P.D., Hes, R.: 1995, A\&AS 114, 259

de Vries, W.H., Barthel, P.D., O’Dea, C.P.: 1997, A\&A 321, 105 